\title{
Parents' perceptions of personal utility of exome sequencing results
}

\author{
Lonna Mollison, $\mathrm{PhD}{ }^{1}{ }^{1}$, Julianne M. O’Daniel, MS, CGC ${ }^{1}$, Gail E. Henderson, $\mathrm{PhD}^{2}$, \\ Jonathan S. Berg, MD, $\mathrm{PhD}^{1}$ and Debra Skinner, $\mathrm{PhD}^{3}$
}

\begin{abstract}
Purpose: Clinical genome or exome sequencing (GS/ES) provides a diagnosis for many individuals with suspected genetic disorders, but also yields negative or uncertain results for the majority. This study examines how parents of a child with an undiagnosed condition attribute personal utility to all types of ES results.
\end{abstract}

Methods: Return of 31 exome sequencing results was observed during clinic sessions, followed by semistructured interviews with parents one month later. Observations and interviews were recorded and transcribed. Data display matrices were used for content analysis and systematic comparisons of parents' perceptions of utility.

Results: ES results could not provide all the answers to parents' questions, especially in cases of clinically uninformative results, but parents nonetheless attributed utility to the knowledge gained. Parents across all results categories used the genomic information to rule out possible causes, end or postpone the diagnostic odyssey, and shift focus to treatment and management of symptoms.

Conclusion: This study suggests that parents value even uninformative ES results while expressing hope for future discoveries. As pediatric genetics moves toward GS/ES as a firsttier test, how parents perceive the personal utility of negative or uncertain results is an important topic for genetic counseling and further research.

Genetics in Medicine (2020) 22:752-757; https://doi.org/10.1038/s41436019-0730-8

Keywords: exome sequencing; diagnostic testing; pediatrics; parent perceptions; personal utility

\section{INTRODUCTION}

Advancements in DNA sequencing technology enable efficient analysis of the human genome or exome (a target consisting of primarily protein-coding portions). This ability has proven to be clinically useful in monogenic disease. ${ }^{1}$ Studies have reported a diagnostic yield for exome sequencing that ranges from $25 \%$ to $40 \%$ of sequenced patients depending on the presenting phenotypes. ${ }^{2}$ For individuals with suspected genetic disorders and their clinicians, identifying the molecular cause is a quintessential quest. Even though a genetic diagnosis often will not lead to a cure or even clinical alterations in day-to-day symptom management or health outcomes, ${ }^{3}$ it is valued for ending the diagnostic journey, reducing its associated emotional and financial burdens, and potentially providing information relevant to making reproductive decisions and accessing appropriate services and supports. ${ }^{4-6}$

Many of the patients referred for sequencing are children who have developmental disabilities of suspected genetic origin. ${ }^{7,8}$ Often their caregivers have sought specialty care to answer five key questions: What is the condition called? How did it happen? What will the future hold? Is there a treatment or a cure? Who else is at risk? ${ }^{9}$ Clinical genome or exome sequencing (GS/ES) may answer some or all of these questions, but for a majority of patients, it will likely yield no or only partial information. In the case of a positive result, parents learn the designation of a specific pathogenic variant, the implicated gene, and possibly the name of an associated syndrome. They may also learn the mode of inheritance and who else may be at risk. Although a cure is unlikely, they may gain information relevant to prognosis or treatment. Positive results thus provide at least partial answers to the five questions, but what are the value and utility for caregivers who learn a negative or uncertain result? As pediatric genetics moves more toward GS/ES as a first-tier test, understanding how parents respond to all types of ES results is an important research agenda. ${ }^{10,11}$

Researchers have defined dimensions of personal utility, ${ }^{12}$ and extrapolated what these may be from studies of various types of genetic testing, ${ }^{13}$ but to date there is limited information on participants' actual responses to GS/ES results, especially results that are uncertain or negative. The few studies that exist report how parents of children who had undergone GS/ES valued a positive result but were ambivalent about uncertain results, ${ }^{14}$ hoped for more information in the future, ${ }^{15}$ and valued ES information in pediatric cancer for its psychological benefits and pragmatic uses. ${ }^{16}$

${ }^{1}$ Department of Genetics, University of North Carolina at Chapel Hill, Chapel Hill, NC, USA; ${ }^{2}$ Department of Social Medicine, University of North Carolina at Chapel Hill, Chapel Hill, NC, USA; ${ }^{3}$ FPG Child Development Institute, University of North Carolina at Chapel Hill, Chapel Hill, NC, USA. Correspondence: Lonna Mollison (lmolliso@email.unc.edu) 
This study contributes to this nascent literature, filling in the gap of how personal utility may be perceived from even negative or uncertain results. As part of the North Carolina Clinical Genomic Evaluation by NextGen Exome Sequencing (NCGENES) research study, ${ }^{17}$ we conducted a systematic examination of 31 parents' attributions of personal utility to all types of ES results, positioning their responses in the context of clinicians' communications. Our findings indicate that although ES did not provide all the answers to parents' key questions, especially in cases of clinically uninformative results, they nonetheless valued and used the knowledge gained through ES in nuanced ways that could inform genetic education and counseling.

\section{MATERIALS AND METHODS}

NCGENES was one of nine research projects funded by the National Institutes of Health/National Human Genome Research Institute (NIH/NHGRI) to identify and address the scientific, clinical, and ethical challenges of using sequencing technology in clinical medicine. ${ }^{18,19}$ Pediatric patients with intellectual disability, congenital malformations, or ophthalmological disorders suspected of having genetic etiologies were referred to NCGENES by their clinicians. Primary aims of NCGENES were to evaluate how well ES performed for clinical diagnostic use and to assess participants' understandings of and responses to ES. To address this latter aim, researchers trained in participant observation and interview methods observed clinic sessions in which NCGENES clinician-researchers returned ES results, and interviewed parents of child patients who participated in these sessions.

Of the 222 children enrolled in NCGENES, 38 obtained positive results, 130 had negative results, and 54 had uncertain results. We approached parents of children from each of these categories for permission to observe the return of results clinic visit and interview them four weeks later. We purposively selected the sample to include at least ten parents for each results category who were diverse in ethnicity and education. Research assistants (RAs) first called parents to explain the study before the clinic visit and mailed consent forms to those who were interested in participating (parents did not know the result before attending the clinic visit). Forty-six families were approached to obtain the sample of 31 . Two families could not be reached, ten declined, and three did not attend the scheduled clinic visit. The RA obtained written consent from parent participants before observing and audio recording the clinic session. For 15 of the 31 sessions observed, both parents attended. In 2 cases, only the father attended and in 14 cases, only the mother. Approximately four weeks later, an RA conducted a semistructured interview by telephone with one parent. For the 15 cases in which both parents attended, we allowed them to decide which one would do the interview. Questions were designed to elicit parents' understandings of clinicians' communications and the ES results, and how they evaluated and used the information (see Supplemental Material). Clinic observations and interviews lasted about one hour each, and were audio recorded and transcribed verbatim.
The University of North Carolina at Chapel Hill institutional review board (IRB) approved all procedures and protocols under IRB 11-1865.

For this study, the analysis focused specifically on parents' perceptions of the personal utility of the ES result, even if clinically uninformative. Two authors (L.M. and D.S.) conducted a systematic content analysis of transcripts of the return of results sessions and the parent interviews, ${ }^{20}$ employing data display matrices (a visual display of content analysis) to summarize and categorize parents' responses related to their perceptions of utility of the ES result. ${ }^{21} \mathrm{We}$ then examined similarities and differences of responses within and across the three categories of results.

\section{RESULTS}

\section{Participant characteristics}

Sociodemographics of the 31 parents interviewed are depicted in Table 1. Participants were predominately non-Hispanic white and female with $45 \%$ having a college degree or higher. Child patients ranged in age from $<1$ year to 16 years.

\section{The clinic context of parents' responses}

NCGENES clinicians' communications about positive and negative results were fairly uniform. When returning positive results, they focused primarily on known genetic and clinical information related to cause, prognosis, treatment, and risks for other children or relatives. In the case of negative results, clinicians began the session by informing parents that ES had not found the cause of their child's condition and delineated the reasons why this might be the case: limitations of the technology, limited current knowledge, and the possibility that the condition did not have a genetic etiology. Although clinicians could provide little information related to parents' key questions, they did address causality by explaining that

Table 1 Demographic descriptions of parents.

\begin{tabular}{lll} 
& $\begin{array}{c}\text { All parents } \\
(n=22)\end{array}$ & $\begin{array}{l}\text { Parents in study } \\
(n=31)\end{array}$ \\
\hline Gender & & \\
\hline Male & $9.9 \%(22)$ & $12.9 \%(4)$ \\
\hline Female & $87.8 \%(195)$ & $87.1 \%(27)$ \\
\hline Left blank & $2.3 \%(5)$ & 0 \\
\hline Race & & \\
\hline Non-Hispanic White & $78.4 \%(174)$ & $90.3 \%(28)$ \\
\hline African American & $9.5 \%(21)$ & $3.2 \%(1)$ \\
\hline American Indian & $1.3 \%(3)$ & 0 \\
\hline Asian & $1.8 \%(4)$ & $6.5 \%(2)$ \\
\hline Other/left blank & $9 \%(20)$ & 0 \\
\hline Education & & \\
\hline High school 12 years or less & $24.3 \%(54)$ & $3.2 \%(1)$ \\
\hline Some college/associate's degree & $39.6 \%(88)$ & $51.6 \%(16)$ \\
\hline College degree & $22.5 \%(50)$ & $16.2 \%(5)$ \\
\hline Advanced degree & $11.3 \%(25)$ & $29 \%(9)$ \\
\hline Left blank & $2.3 \%(5)$ & 0 \\
\hline
\end{tabular}


ES had ruled out numerous genes that might have been associated with the child's condition, and in some cases, they talked about a decreased likelihood that the cause was genetic.

Clinicians' communication of uncertain results was more varied, depending on the degree of uncertainty. ${ }^{10}$ They began the session with a statement such as "we may have found the answer," and then expanded on the reasons why they could not be certain that the discovered variant was the cause: (1) the identification of a novel variant of uncertain significance in a gene highly associated with the child's phenotype, (2) identification of a pathogenic/likely pathogenic variant in a gene that does not seem to align perfectly with the presenting phenotypes, or (3) identification of a variant(s) in a gene that is associated with multiple conditions or variable phenotypes, making it difficult to determine which condition is clinically relevant. In some cases, family testing was recommended for potentially providing more certain answers.

No matter what the result, some degree of "future-making" was part of the conversation, that is, the field of diagnostic genomics is in its infancy and more research will likely produce new discoveries. For example, clinicians could provide little information on what the future might hold for those with a positive diagnosis of a newly described condition, but they communicated that ongoing research would likely lead to more knowledge of genotype-phenotype associations and perhaps new treatments. For those with negative or uncertain results, clinicians informed parents of future plans to reanalyze their child's ES data if relevant information emerged. This included reexamining variants for pathogenicity, analyzing other diagnostic gene lists, and conducting research sweeps to identify new genes and variants associated with the child's reported phenotype.

Clinicians also thanked parents for their contributions to the research enterprise, noting that they were in this together. They urged parents to contact them with any new information about genes or their child that could guide a reinterpretation, as reflected in this exchange with a mother who learned a negative result for her child with seizures and intellectual disability:

Clinician: If you're interested in searching for what might be the cause, and you might come across a condition that you think fits it pretty well, and you might want to look and see was that gene on here [the research report], and if you or his physician say, "Gosh. You really should have looked at this gene because it really fits his picture." And it wasn't on any of our lists. Well, we should add it to our list and then look at it. Right?

Mother: Yeah.

Clinician: So we would definitely welcome feedback on genes that should have been looked at so that we can add them to our list and look at them.
While endorsing this kind of continued partnership, clinicians at the same time indicated that the future application of emerging technology and research, reanalysis, and reinterpretation was largely in their purview as reflected in this clinician's promise:

As more and more is learned about different genes...those genes will get added to our lists, and we can reanalyze the data, and we'll do so on people who have uncertain results or negative results. And it could be that a much more compelling explanation is found next year or the year after that, and that is to take into account the fact that we're learning new things on literally a daily basis.

\section{Parents' perceptions of personal utility of positive diagnostic results}

Parents' reactions to a positive ES result were similar to what has been reported in numerous studies of parents' responses to a positive diagnosis from any type of test. ${ }^{16,22,23}$ Some felt absolved for having caused the condition (e.g., "It calms me to know and realize the doctors didn't do anything wrong. I didn't do anything wrong. It's just something that happened that couldn't be prevented"-Mother of a son with a FOXG1 variant). Parents also expressed relief at being able to stop their search for a diagnosis as ES produced a result when other tests failed to do so (e.g., "It's just comforting....it's great to be able to stop with the genetic testing... and be able to stop that particular journey"-Mother of a son with Coffin-Siris syndrome). This sense of relief was often countered, however, by the uncertainties of how a rare disease would progress (e.g., "Well, I mean it's a relief that I know what the name is, but it's worrying because what's her future like?"-Mother of a daughter found to have a form of Charcot-Marie-Tooth disease).

For these parents, the diagnostic odyssey ended but because a diagnosis did not change treatment in most cases, the therapeutic journey began. ${ }^{14,15}$ They echoed clinicians' predictions that ongoing genomic research and informationsharing in the future would lead to treatments for rare diseases. For example, a mother who learned her daughter's seizures were caused by a deletion in chromosome 16, for which little is currently known, said, "Something may change in the future where we would have more information on it... and more ways to maybe treat it."

\section{The personal utility of a negative result}

Parents expressed hope that ES would identify the genetic cause of their child's condition, but they did not have high expectations of this happening because of the information provided in the consent process. Although somewhat disappointed in the negative result, the ability of ES to rule out hundreds of possible genes that could cause the condition was seen as informative and even "good news" to some parents. As a father whose son has microcephaly explained: 
I guess it's a relief to know they didn't find anything, which is-I guess it's a good news, bad news thing. They didn't find anything, which you wish they would have, but of the many things they could have found they didn't, which means he doesn't have those particular reasons or whatever aren't the cause, which can be good too.

Parents also indicated that one valuable outcome of learning a negative result from ES was that it allowed them to pause the diagnostic odyssey and turn their attention more to symptom management. A father of a son with motor delays, cardiovascular anomaly, and microcephaly reflected: "I think we've perhaps resigned ourselves to the fact that we may never know, and so we have to move forward in treating the symptoms of his condition and how they manifest themselves rather than worrying about what the condition actually is."

Parents talked about their experience of undergoing ES as having "done all they could," "trying everything" to find a genetic cause. They had used the latest technology and saw no further avenue to explore, at least at the time, effectively ending their diagnostic odyssey and the burden of being responsible for this quest. A mother whose daughter had multiple health concerns (microcephaly, developmental delays, poor growth, arrhythmia, gastric emptying delay) said, "We have to kind of give up. Not give up on her, but give up on finding out why. So I felt like the doctor really kind of gave me permission to stop looking." Another mother of a girl with congenital malformations said, "We're just going to have to be at peace that we're not going to have an answer. So for us it was a big deal because it was kind of like the final hurrah. Like no more hospitals, no more testing. This was it for us."

These parents thus recognized that if ES could not reveal the cause, the likelihood of finding an answer in the near future was improbable. A negative result, though perhaps only contingent, gave parents temporal closure to their search, but they reflected the hope voiced by the clinicians that future discoveries could benefit their child (e.g., "We're hopeful that sooner rather than later somewhere in the world all these genetic doctors, that looking back at us...hopefully as they learn more and more they will be able to put the puzzle together."-Mother of a daughter with seizures, developmental delay, and other conditions).

Although parents did not have a definitive diagnosis that provided entrée to social and medical networks, some parents nevertheless used the negative result to share their experience of ES and connect with others. A mother of a daughter who has seizures said she shared the ES results on her child's Facebook page, writing, "We got the results of the genetic testing, and there's no diagnosis to this point, but she's in the study for three more years, and they may be able to interpret results differently then." Another mother of a son with intellectual disability (ID)/autism said she used what she had learned about other diseases discussed during the clinic study visit: "Even though he doesn't have a Cornelia de Lange diagnosis, when that was mentioned I started doing research about it and actually have connected with several parents through support groups and Facebook."

\section{Finding personal utility in uncertainty}

Parents' perceptions of the value and usefulness of uncertain results reflected the degree of uncertainty conveyed by clinicians. For some, the uncertain result was most likely definitive and thus comparable with a definitive positive result. In one such case, clinicians told parents that a novel variant identified in the $B B S 10$ gene was a likely cause for their child's Bardet-Biedl syndrome. The parents used this result to locate support groups and enroll in a new research study. Parents who learned a result that was more uncertain viewed it as "a place to start" or something to keep in mind, but not to act upon until more was known. As a mother of a son with hearing loss said, "We can't do much about it, but it's an interesting data point that at some point could mean something and have more impact."

A number of complexities compound the uncertainties that arise from ES, including the likelihood that more complex forms of inheritance, such as oligogenic or polygenic, may prove more common than anticipated. For example, a mother whose son had retinitis pigmentosa (RP) expressed her uncertainty about his ES results (heterozygous pathogenic variants in three different recessive genes related to RP):

They identified three genes, defective genes that they know -they're known to link to this disease.... So they didn't know if it could be possibly a new pairing of two of the three that are causing it, all three of them causing it, or if there's one of them causing it, and they just didn't find the other one, that they just missed it somehow.

In cases such as this, NCGENES clinicians encouraged families to continue to work with them in a partnership that could eventually resolve uncertainty. Parents understood that they were participating in a research project for the purpose of building the knowledge base. The mother of the son with RP said, "So they're basically saying that we may-that [our child] may be actually able to teach them something, which is good in one way." Another mother of a son with ID/autism said, "Let's figure this out. I'm hoping the more information that we get like maybe one day they'll be able to help...maybe not my son, but maybe somebody else's kid like five, ten years down the road."

\section{Secondary findings}

A powerful yet controversial aspect of ES is its ability to identify pathogenic variants for conditions unrelated to the clinical presentation. ${ }^{24-26}$ NCGENES was designed to return medically actionable secondary findings along with diagnostic results. ${ }^{2}$ Although unrelated to the presenting phenotypes, secondary findings provided parents with information on conditions that had available treatments, screening, and interventions. Seven children of the total pediatric sample of 222 in NCGENES had such secondary findings; three were 
interviewed as part of this study. All three had findings related to blood clotting disorders. Similar to other reports, ${ }^{27}$ parents in NCGENES who received this information viewed it as valuable and did not express regret or distress at having learned it. A mother whose child had a negative diagnostic result for congenital neutropenia learned of a positive secondary result for a pathogenic variant in $P R O C$, a gene responsible for making protein $\mathrm{C}$, involved in controlling blood clotting. She responded to this information, saying:

You know we were actually hoping for some kind of answer with her neutropenia, but I mean unfortunately we didn't find any answers.... The good thing is we did find something else that she does have, so that is now in her medical record. So I'm glad to know what else was going on with her.

\section{DISCUSSION}

This study examined how parents attribute personal utility to ES results, even if clinically uninformative. One important finding is that ES effectively ended the diagnostic journey of not only parents who learned positive results, but also those with negative results. Parents with uncertain results also ceased their journey when no further action such as familial testing was warranted. As ES was the most advanced and comprehensive genetic test available at the time, parents without definitive results felt they had done all they could. They effectively ended their own search for a diagnosis, waiting on clinicians to inform them of any future discoveries that might rekindle the quest. This is in stark contrast to prior studies conducted before the advent of genome sequencing that indicated parents felt compelled to continue their search for a genetic diagnosis when pediatric genetics did not provide an answer. ${ }^{28}$ Using GS/ES as a first-tier test could save parents from the financial and emotional costs associated with continued searching, even in cases of negative or uncertain results. $^{29,30}$

Dimensions of personal utility may include how individuals value their participation in research even if not reaping the benefits immediately. ${ }^{12,13}$ Advanced sequencing technologies have led to new gene-disease associations and enhanced diagnostic yield, but have also outpaced the ability to translate that knowledge into novel treatments and cures. This undeniable tension between technological capability and clinical utility was handled remarkably well by parents whose children underwent ES, perhaps due to the NCGENES clinicians' reassurances that more knowledge would be forthcoming in the future. Some parents anticipated discoveries within the short time span of the study. For others, this knowledge existed in a more nebulous future. For all categories of results, parents had a keen ability to appreciate the novelty of ES and look toward a future when accumulated knowledge would benefit their own or other children.

Findings from this study have implications for genetic counseling and education. A diagnostic result from any type of test requires that parents digest what the information means within the context of their lives, the ongoing care of their child, and potential reproductive choices. For these reasons, it is important that pretest counseling prepare parents with realistic, limited expectations about what GS/ES can and cannot provide, and the likelihood that GS/ES will not result in a definitive genetic diagnosis or provide answers to all of their questions. ${ }^{31-34}$ Even so, given the findings of this study, pretest counseling could indicate that even negative or uncertain results may provide information of some value and usefulness to parents, such as ending or transferring the search to clinicians, participating in research that could benefit their child or other children, or providing some knowledge that could lead to networking with other parents.

One limitation of this study is that it was conducted as a research project relatively early in the application of GS/ES, and some of the findings may not be reproduced in a purely clinical setting where parents may have higher expectations for answers. Furthermore, in situations where future-making may not be as large a part of the interchange between clinician and patient, those responses may vary. Additionally, the selection criteria for participants in the study (patients who were already undergoing a diagnostic odyssey whose physicians thought they might benefit from ES) may overrepresent parents who were motivated to seek such information, and thus bias our portrait of responses to ES results. Finally, although we attempted to achieve ethnic and racial diversity in our sample, nine families we approached for recruitment but who declined were from underrepresented minority groups. This limitation reflects barriers to participation in the larger NCGENES project, ${ }^{19}$ and curtails our ability to represent any differences in how ethnically and racially diverse parents might view the personal utility of ES.

Despite these limitations, this study contributes to the systematic examination of responses to GS/ES, providing useful insights for providers into how parents contextualize and use their child's diagnostic GS/ES results, even if uninformative. It also portrays clinicians' roles in these evaluations such as promises to take on the responsibility for continued searches for a diagnosis. Further, it emphasizes the importance of building realistic hopes for future discoveries and ongoing partnerships between parents and clinicians in this endeavor. Future research could examine parents' responses to sequencing results in purely clinical contexts to assess their evaluations outside of a research context, and how clinical-family partnerships continue or evolve in light of new discoveries.

\section{SUPPLEMENTARY INFORMATION}

The online version of this article (https://doi.org/10.1038/s41436019-0730-8) contains supplementary material, which is available to authorized users.

\section{ACKNOWLEDGEMENTS}

The research on which this study is based was funded by the National Human Genome Research Institute (NHGRI) of the 
National Institutes of Health $(\mathrm{NIH})$ under award number U01 HG006487-01; the Center for Genomics and Society at the University of North Carolina-Chapel Hill, funded by the NHGRI/ $\mathrm{NIH}$ under award number 2P50HG004488; and TraCS Institute from the Clinical and Translational Science Award program of the National Center for Advancing Translational Sciences, National Institutes of Health under award number 1UL1TR001111. The content is solely the responsibility of the authors and does not necessarily represent the official views of the $\mathrm{NIH}$.

\section{DISCLOSURE}

The authors declare no conflicts of interest.

Publisher's note Springer Nature remains neutral with regard to jurisdictional claims in published maps and institutional affiliations.

\section{REFERENCES}

1. Van Dijk EL, Auger $H$, Jaszczyszyn $Y$, Thermes $C$. Ten years of nextgeneration sequencing technology. Trends Genet. 2014;30:418-426.

2. Strande NT, Berg JS. Defining the clinical value of a genomic diagnosis in the era of next-generation sequencing. Annu Rev Genomics Hum Genet. 2016;17:303-332

3. Stivers T, Timmermans $S$. The actionability of exome sequencing testing results. Sociol Health IIIn. 2017;39:1542-1556.

4. Sawyer SL, Hartley T, Dyment DA, et al. Utility of whole-exome sequencing for those near the end of the diagnostic odyssey: time to address gaps in care. Clin Genet. 2016;89:275-284.

5. Normand EA, Alaimo JT, Van den Veyver IB. Exome and genome sequencing in reproductive medicine. Fertil Steril. 2018;109:213-220.

6. Sapp JC, Dong D, Stark C, et al. Parental attitudes, values, and beliefs toward the return of results from exome sequencing in children. Clin Genet. 2014:85:120-126.

7. Valencia CA, Husami A, Holle J, et al. Clinical impact and cost-effectiveness of whole exome sequencing as a diagnostic tool: a pediatric center's experience. Front Pediatr. 2015;3:67.

8. Berg AT, Coryell J, Saneto RP, et al. Early-life epilepsies and the emerging role of genetic testing. JAMA Pediatr. 2017;171:863-871.

9. Berg JS. Genome-scale sequencing in clinical care: establishing molecular diagnoses and measuring value. JAMA. 2014;312:1865-1867.

10. Skinner D, Roche MI, Weck KE, et al. "Possibly positive or certainly uncertain?": participants' responses to uncertain diagnostic results from exome sequencing. Genet Med. 2017;20:313-319.

11. Skinner $D$, Raspberry KA, King $M$. The nuanced negative: meanings of a negative diagnostic result in clinical exome sequencing. Sociol Health IIIn. 2016;38:1303-1317.

12. Kohler JN, Turbitt $\mathrm{E}$, Lewis $\mathrm{KL}$, et al. Defining personal utility in genomics: a Delphi study. Clin. Genet. 2017;92:290-297.

13. Kohler JN, Turbitt E, Biesecker BB. Personal utility in genomic testing: a systematic review. Eur J Hum Genet. 2017;25:662-668.

14. Krabbenborg L, Vissers LE, Schieving J, et al. Understanding the psychosocial effects of WES test results on parents of children with rare diseases. J Genet Couns. 2016;25:1207-1214
15. Rosell AM, Pena LD, Schoch K, et al. Not the end of the odyssey: parental perceptions of whole exome sequencing (WES) in pediatric undiagnosed disorders. J Genet Couns. 2016;25:1019-1031.

16. Malek J, Slashinski M, Robinson JO et al. Parental perspectives on wholeexome sequencing in pediatric cancer: a typology of perceived utility. JCO Precision Oncology 2017. Downloaded from ascopubs.org on October 7, 2019 from 045.037.116.214.

17. Foreman LeeK, Evans J. The NC Genes Project: exploring the new world of genomic sequencing. NC Med J. 2012;74:500-504.

18. Green R, Goddard K, Jarvik G, et al. Clinical sequencing exploratory research: accelerating evidence based practice of genomic medicine. Am J Hum Genet. 2016;98:1051-1066.

19. Moore $\mathrm{E}$, Roche $\mathrm{M}$, Rini $\mathrm{C}$, et al. Examining the cascade of participation in a genomic medicine research study: barriers and facilitators to achieving diversity. Public Health Genomics. 2017;20:332-342.

20. Charmaz K. Constructing grounded theory: a practical guide through qualitative analysis. London: Sage; 2006.

21. Miles MB, Huberman AM, Saldaña J. Qualitative data analysis: a methods sourcebook. 3rd ed. Los Angeles: Sage; 2013.

22. Reiff M, Bugos E, Giarelli E, et al. "Set in stone" or "ray of hope": parents' beliefs about cause and prognosis after genomic testing of children diagnosed with ASD. J Autism Dev Disord. 2017;47: 1453-1463.

23. Raspberry K, Skinner D. Enacting genetic responsibility: experiences of mothers who carry the fragile $X$ gene. Sociol Health IIIn. 2011;33: 420-433.

24. Berg JS, Khoury MJ, Evans JP. Deploying whole genome sequencing in clinical practice and public health: meeting the challenge one bin at a time. Genet Med. 2011;13:499-504.

25. Roche M, Berg JS. Incidental findings with genomic testing: implications for genetic counseling practice. Curr Genet Med Rep. 2015;3:166-176.

26. Berg JS, Foreman A, O'Daniel J, et al. A semiquantitative metric for evaluating clinical actionability of incidental or secondary findings from genome-scale sequencing. Genet Med. 2016;18:467-475.

27. Hart MR, Biesecker BB, Blout CL, et al. Secondary findings from clinical genomic sequencing: prevalence, patient perspectives, family history assessment, and health-care costs from a multisite study. Genet Med. 2018;20:1-9.

28. Schaffer R, Kuczynski KJ, Skinner D. Producing genetic knowledge and citizenship through the internet: mothers, pediatric genetics, and cybermedicine. Sociol Health IIIn. 2008;30:145-159.

29. Stark Z, Tan TY, Chong B, et al. A prospective evaluation of whole-exome sequencing as a first-tier molecular test in infants with suspected monogenic disorders. Genet Med. 2016;18:1090-1096.

30. Scocchia A, Wigby KR, Masser-Frye $D$, et al. Clinical whole genome sequencing as a first-tier test at a resource limited dysmorphology clinic in Mexico. NPJ Genom Med. 2019;4:5.

31. Biesecker $B$, Klein $W$, Lewis $K L$, et al. How do research participants perceive "uncertainty" in genomic sequencing? Genet Med. 2014;16: 977-980.

32. Stivers T, Timmermans S. Negotiating the diagnostic uncertainty of genomic test results. Soc Psychol Q. 2016;79:199-221.

33. Amendola LM, Lautenbach D, Scollon S, et al. Illustrative case studies in the return of exome and genome sequencing results. Per Med. 2015; $12: 283-295$.

34. Bernhardt $B$, Roche $M$, Perry $D$, et al. Experiences with obtaining informed consent for genomic sequencing. Am J Med Genet A. 2015; 167A:2635-2646. 